\title{
Effects of Serum from a Fibrodysplasia Ossificans Progressiva Patient on Osteoblastic Cells
}

\author{
Itoko Hisa $^{1}$, Akira Kawara ${ }^{2}$, Takenobu Katagiri ${ }^{3}$, Toshitsugu Sugimoto ${ }^{4}$, Hiroshi Kaji ${ }^{15,6 *}$ \\ ${ }^{1}$ Division of Diabetes and Endocrinology, Department of Internal Medicine, \\ Kobe University Graduate School of Medicine, Kobe, Japan \\ ${ }^{2}$ Kawara Clinic, Kobe, Japan \\ ${ }^{3}$ Division of Pathophysiology, Research Center for Genomic Medicine, Saitama Medical University, Japan \\ ${ }^{4}$ Internal Medicine 1, Shimane University Faculty of Medicine, Izumo, Japan \\ ${ }^{5}$ Division of Cellular and Molecular Medicine, Department of Physiology and Cellular Biology, \\ Kobe University Graduate School of Medicine, Kobe, Japan \\ ${ }^{6}$ Department of Physiology and Regenerative Medicine, Kinki University Faculty of Medicine, Osaka, Japan \\ Email: "hkaji@med.kindai.ac.jp
}

Received 5 December 2011; revised 1 January 2012; accepted 30 January 2012

\begin{abstract}
Introduction: Fibrodysplasia ossificans progressiva (FOP) is a rare autosomal dominant disorder of skeletal malformations and progressive extraskeletal ossification. Patients and Methods: In the present study, we present a case of FOP with marked progressive ossification of extraskeletal tissues. We investigated whether soluble factors in serum would affect the osteoblast phenotype by examining the effects of serum from this patient and control subjects on mouse osteoblastic MC3T3-E1 cells. Results: The clinical findings of this patient were compatible with FOP, and direct sequence analysis of genomic DNA demonstrated the presence of a heterozygous 617G > A (R206H) mutation of activin type 1 receptor (ACVR1). Serum from the FOP patient enhanced the level of alkaline phosphatase (ALP) in Western blotting, compared with serum from the control, in MC3T3-E1 cells. Moreover, serum from the FOP patient enhanced the levels of ALP, osteocalcin and bone morphogenetic protein-2 mRNA in these cells. Conclusion: We presented a case of FOP with progressive ossification in extra-skeletal tissues with ACVR1 mutation. The present data suggest that the serum from this patient includes some soluble factors, which might enhance the osteoblast differentiation and BMP2 expression in mouse osteoblastic cells.
\end{abstract}

Keywords: Fibrodysplasia Ossificans Progressiva; Osteoblast; Bone Morphogenetic Protein

\section{Introduction}

Fibrodysplasia ossificans progressiva (FOP) is a rare autosomal dominant disorder of skeletal malformations and progressive extraskeletal (heterotopic) ossification, characterized by congenital malformation of the great toes. Heterotopic ossification of the muscles, tendons, ligaments, and fascia begins in childhood and can be induced by trauma or for no reason, leading to extraarticular ankylosis of all major joints in the axial and appendicular skeleton, which renders movement impossible $[1,2]$. Several reports suggest that the earliest pathological finding in FOP is perivascular lymphocytic infiltration into normal-appearing skeletal muscle, followed by muscle-cell degeneration and highly vascular fibroproliferative soft tissue swelling. Fibroproliferative lesions evolve through an endochondral process into mature lamellar bone with marrow elements [2,3].

*Corresponding author.
As for the pathogenesis of FOP, Shafritz et al. [4] reported that the ectopic osteogenesis of FOP is associated with increased levels of bone morphogenetic protein (BMP)-4 in lymphocytes, and Olmsted et al. [5] further showed that the rate of BMP-4 transcription was elevated in cells of FOP patients. Moreover, de la Pena et al. [6] reported that lymphocytes in FOP exhibited a defect in BMP receptor type IA (BMPR-IA) internalization and increased the activation of downstream signaling, suggesting that altered BMP receptor trafficking underlies ectopic bone formation in FOP. Recently, Shore et al. [7] mapped FOP to chromosome 2q23-24 by linkage analysis and identified an identical heterozygous mutation (617G $>$ A; R206H) in the glycine-serine (GS) activation domain of activin A type I receptor gene (ACVR1). Protein modeling induces destabilization of the GS domain, consistent with constitutive activation of ACVR1, expressed in many tissues, including skeletal muscle and chondrocytes. Constitutive activation of ACVR1 induces al- 
kaline phosphatase (ALP) activity in C2C12 cells and upregulates BMP-4 [7]. Moreover, BMP-4 induces constitutive activation of ACVR1 in these cells [8]. These findings indicate that ACVR1 is a strong candidate gene for FOP. Although the causal gene of FOP was found, numerous points in the pathogenesis of FOP remain unclear. Moreover, there have not been any reports about the pathological role of serum from the patient with FOP to our knowledge.

In the present study, we present a case of FOP with marked progressive ossification of extraskeletal tissues. This patient had known ACVR1 mutation, and serum from the patient possessed activity that modulated the osteoblast phenotype.

\section{Patients and Methods}

\subsection{Patient}

A 36-year-old man had progressive ectopic ossification. Short thumbs and malformed big toes were noted at birth. Psychomotor epilepsy occurred at 1 year old and scoliosis was noted at 4 years old. The first ossification appeared on his back at 6 years old and rapidly formed new ossification in the biopsy area, the first ossification area and other normal skin area; therefore, he was diagnosed with FOP at 6 years old. Ossification and joint fixation progressed from the back, neck, hip, and jaw to all limbs. At 36 years old, he is bedridden in the prone position, because almost all of his joints and back muscles have been fixed by ossification. No close relatives show FOP symptoms and there is no parental consanguinity.

His clinical features showed an asymptomatic lump on the left side of his head and along the vertebral column, fixation of the whole body, marked lordosis and scoliosis, bedsores on both sides of the greater trochanter, and malformed big toes. His consciousness was clear and speech was fluent. There was no disorder in mental and pubertal development. Glucocorticoid (betamethasone 0.75 $\mathrm{mg}$ per day) and etidronate (200 mg per day for two weeks every three or four months) have been administered in order to suppress progressive heterotopic ossification since he was 33 years old.

The laboratory findings showed high and low levels of bone resorption and bone formation indices, respectively [bone-type ALP 12.7 U/l [normal range: 9.6 - 35.4], urinary type I collagen N-telopeptides (NTx) $131 \mathrm{nmol}-$ $\mathrm{BCE} / \mathrm{mmolCr}$ [normal range: 9.3 - 54.3], osteocalcin (OCN) $1.8 \mathrm{mg} / \mathrm{l}$ [normal range: 2.5 - 13]. Renal function was not disturbed (creatinine clearance $96.3 \mathrm{ml} / \mathrm{min}$ ). Serum levels of calcium, phosphorus and intact PTH were $2.2 \mathrm{mmol} / \mathrm{l}$ (normal range: 2.10 - 2.48), $3.7 \mathrm{mg} / \mathrm{dl}$ (normal range: 2.4 - 4.5), 51 ng/l (normal range: 10 - 65), respectively. Urinary calcium excretion was increased (302.8 mg/day). Arterial blood gas under room air was also analyzed (pH 7.429, $\mathrm{pCO}_{2} 52.7 \mathrm{mmHg}, \mathrm{pO}_{2} 89.7$ $\mathrm{mmHg}, \mathrm{HCO}_{3}^{-} 34.1 \mathrm{mmol} / \mathrm{l}$, BE $\left.8.0 \mathrm{mmol} / \mathrm{l}\right)$.

On X-ray examination (Figures $\mathbf{1 A}$ and $\mathbf{B}$ ), there were multiple areas of soft tissue ossifications and a recently developed lump on the left side of the cranial bone. The vertebral column showed marked lordosis and scoliosis.

On the bone scintigram (Figure 1C), radioisotope accumulation was highly increased on the left side of the cranial bone and the whole vertebra. Other heterogeneous increased radioisotope accumulation was observed in the limbs, sacroiliac joint, hip joints, and soft tissues of the legs.

On pelvic computed tomography, short and broad femoral necks were observed as well as intramuscular ossification throughout the whole body.

\subsection{Materials and Cell Cultures}

Mouse osteoblastic MC3T3-E1 cells were kindly provided by Dr. H. Kodama (Ohu Dental College, Japan). Human recombinant BMP-2 and anti- $\beta$-actin antibody were from Sigma (St.Louis, MO, USA), and anti-tissue non-specific alkaline phosphatase rabbit monoclonal antibody was from Santa Cruz Biotechnology (Santa Cruz, CA, USA). All other chemicals were of analytical grade.

After informed consent, an overnight fasting serum sample was obtained. Serum was kept at $-80^{\circ} \mathrm{C}$ until used for the experiments. The serum was obtained under similar conditions from age- and sex-matched controls. The study was approved by the ethics review board of Kobe University Hospital.

MC3T3-E1 cells were cultured in $\alpha$-minimal essential medium ( $\alpha$-MEM; containing $50 \mathrm{mg} / \mathrm{ml}$ of ascorbic acid) supplemented with $10 \%$ FBS and $1 \%$ penicillin-streptomycin (Invitrogen, Carlsbad, CA, USA). The medium was changed twice a week.
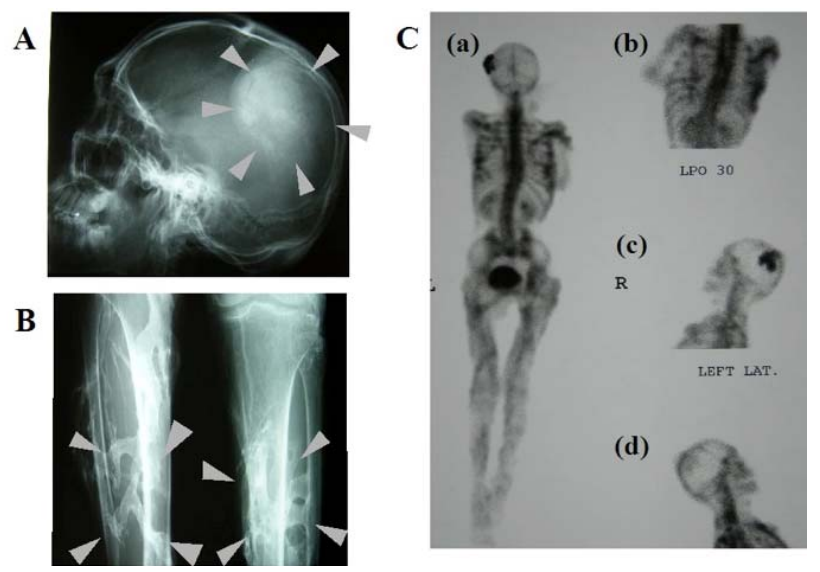

(A) X-ray of skull; (B) X-ray of lower legs; (C) Bone scintigram; images of whole body (anterior-posterior) (a) Thorax (anterior-posterior) (b) and lateral head: right-left (c), left-right (d).

Figure 1. Clinical features of FOP patient. 


\subsection{Protein Extraction and Western Blot Analysis}

After confluent MC3T3-E1 cells were cultured in serumfree $\alpha$-MEM for $12 \mathrm{hr}$, cells were treated with the final $30 \%$ serum from the FOP patient and normal controls for $24 \mathrm{hr}$ before the harvest of cell lysates. In our preliminary study, this time point and serum concentration were the best to observe significant effects of serum from FOP patient in the present study. Cells were lysed with radioimmunoprecipitation buffer with $0.5 \mathrm{mM}$ of phenylmethylsulfonyl fluoride (PMSF), complete protease inhibitor mixture, $1 \%$ Triton $\mathrm{X}-100$, and $1 \mathrm{mM}$ of sodium orthovanadate, as previously described [9]. Cell lysates were centrifuged at $12,000 \mathrm{~g}$ for 20 minutes at $4^{\circ} \mathrm{C}$, and the supernatants were stored at $-80^{\circ} \mathrm{C}$. Protein quantitation was performed with BCA protein assay reagent (Pierce, Rockford, IL, USA). Equal amounts of protein were denatured in SDS sample buffer and separated on $10 \%$ polyacrylamide-SDS gels. Proteins were transferred in $25 \mathrm{nM}$ Tris, $192 \mathrm{mM}$ glycine, and 20\% methanol to polyvinylidene difluoride membranes. Blots were blocked with Tris-buffered saline (20 mM Tris-HCl (pH 7.4) and $137 \mathrm{mM} \mathrm{NaCl}$ ) plus $0.1 \%$ Tween 20 containing 3\% dried milk powder. The membranes were immunoblotted with anti-ALP and $\beta$-actin antibodies. The antigen-antibody complexes were visualized using the appropriate seconddary antibodies (Sigma), and the enhanced chemiluminescence detection system, as recommended by the manufacturer (Amersham Pharmacia Biotech, Buckinghamshire, UK).

\subsection{RNA Extraction and Real-Time PCR}

Total RNA was prepared from cells using TRIZOL reagent. cDNA was synthesized using the SuperscriptTM First-Strand Synthesis System for RT-PCR (GIBCO BRL). Specific mRNA was quantified by real-time PCR using a 7500 Real-time PCR system (Applied Biosystems, Rotkreuz, Switzerland) with SYBR Premix Ex TaqTM II (Perfect Real Time) kits (TaKaRa) according to the manufacture's standard protocol. The mRNA value for each gene was normalized relative to the mouse GAPDH mRNA levels in RNA samples. Primer sequences (forward and reverse) were as follows. GAPDH, 5'-GTGTACATGGTTCCAGTATGAGTCC-3' and 5'AGTGAGTTGTCATATTTCTCGTGGT-3'; OCN, 5'CCTGAGTCTGACAAAGCCTTCA-3' and 5'-GCCGGAGTCTGTTCACTACCTT-3'; ALP, 5'-ATCTTTGGTCTGGCTCCCATG-3' and 5'-TTTCCCGTTCACCGTCCAC-3'; BMP-2, 5'-GGTCACAGATAAGGCATTGC-3' and 5'-GCTTCCGCTGTTTGTGTTTG-3'.

\subsection{Statistics}

All experiments were repeated at least three times with reproducible results. Data are expressed as the mean \pm SEM. Statistical analysis was performed using ANOVA.

\section{Results}

The clinical findings of this patient with progressive extraskeletal osteogenesis induced by physical or surgical trauma were compatible with FOP. Direct sequence analysis of genomic DNA demonstrated the presence of a heterozygous 617G > A (R206H) mutation, which was previously reported [7] (Figure 2). No other family members had the phenotype of FOP and 617G > A (R206H) mutation. These findings confirmed that the diagnosis of this patient was FOP. We investigated whether soluble factors in serum would affect the osteoblast phenotype using MC3T3-E1 cells. We used serum from normal volunteers matched for age and gender as the control.

As shown in Figure 3(a), serum from the FOP patient increased the level of ALP compared with the control serum in MC3T3-E1 cells. Moreover, serum from the FOP patient increased the levels of ALP, OCN and BMP- 2 mRNA in these cells (Figure 3(b)).

\section{Discussion}

BMPs were originally identified because of their ability to induce endochondral bone formation [10]. Although BMPs are synthesized by skeletal cells, their synthesis is

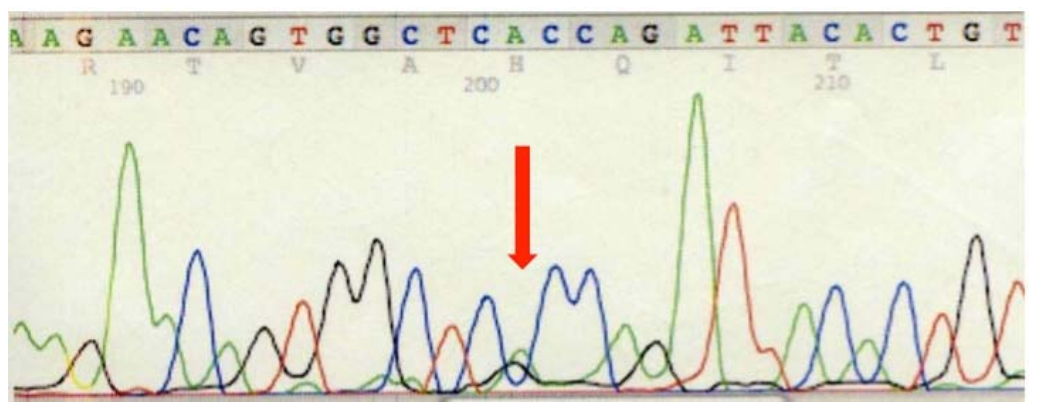

Arrow indicates the mutation site.

Figure 2. Activin A type I receptor gene (ACVR1) analysis. 


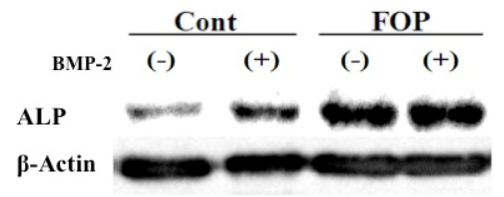

(a)
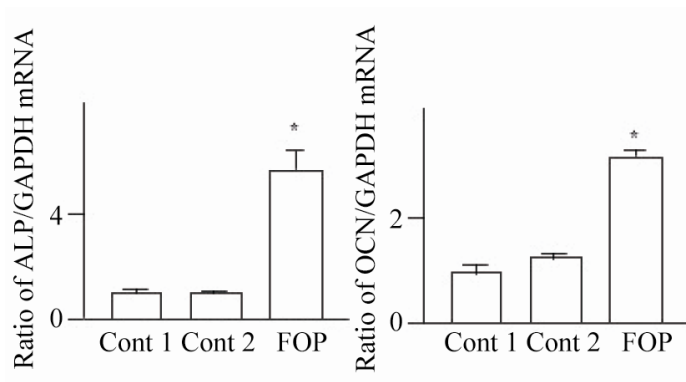

(b)

Figure 3. Serum from FOP affects osteoblast phenotype in MC3T3-E1 cells. (a) After confluent MC3T3-E1 cells were cultured in serum-free $\alpha$-MEM for $12 \mathrm{hr}$, cells were treated with $30 \%$ serum from the FOP patient or a normal control in the presence or absence of $100 \mathrm{ng} / \mathrm{ml}$ BMP-2 for $24 \mathrm{hr}$. Protein extraction and Western blot analysis were then performed for ALP. These data were reproduced three times; (b) After confluent MC3T3-E1 cells were cultured in serum-free $\alpha$-MEM for $12 \mathrm{hr}$, cells were treated with $30 \%$ serum from the FOP patient or a normal control for 24 hr. Total RNA extraction and real-time RT-PCR were then performed for ALP, OCN, BMP or GAPDH mRNA. Data are expressed relative to the GAPDH mRNA value. ${ }^{*} \mathbf{P}<0.01$ relative to serum from normal control 1-treated group.

not limited to bone because they are expressed in a variety of extraskeletal tissues, in which BMPs play a critical role in development and cell function. A fundamental function of BMP-2 and -4 is to induce the differentiation of mesenchymal cells toward osteoblastic linage cells. Furthermore, these BMPs induce endochondral ossification and chondrogenesis in extra-skeletal tissues. Overexpression of BMP-2 and -4 in developing limbs induces an increase in chondrocytes cell number and matrix cartilage, leading to joint fusion $[10,11]$.

As previously reported, the constitutive activation of ACVR1 is considered to play the main role in the pathogenesis of FOP [7]. In this patient with FOP, the reported mutation was identified. This mutation was considered to be the main pathogenesis of ectopic progressive ossification in this patient. FOP lymphocytes expressed 6-fold higher levels of BMP receptor type IA (BMPR-IA) on the cell surface and displayed a marked reduction in ligand-stimulated internalization and degradation of BMPRIA in response to BMP-4. The disrupted BMP signaling pathway caused inappropriate enhancement of the expression of BMP-4 mRNA and protein, resulting in heterotopic ossification [6,7]. From these findings, we can speculate that the heterozygous $617 \mathrm{G}>\mathrm{A}(\mathrm{R} 206 \mathrm{H}) \mathrm{mu}-$ tation in this patient might have induced inappropriate enhancement of the expression of BMP-4 and heterotopic ossification.

In the present study, serum from the FOP patient enhanced the level of ALP on Western blotting of mouse osteoblastic cells, compared with the control serum. Moreover, serum from the FOP patient enhanced the levels of ALP and OCN mRNA in these cells, compared with the control serum. These findings indicate that soluble factors in the serum from the FOP patient were responsible for a stimulation of differentiation in osteoblast. Moreover, we revealed that serum from the FOP patient enhanced the levels of BMP-2 mRNA in these cells, compared with the control serum. Since BMP-2 induces ectopic bone and cartilage formation in extraskeletal tissues in vivo and activates AVCR1, the present data suggest the possibility that there might be some serum soluble factors, which enhancing muscle ossification in the FOP patient.

Bone resorption and bone formation indices are increased and decreased in this FOP patient. The use of glucocorticoid and long-term immobilization might enhance bone resorption. The direct action of glucocorticoid on the gene expression in skeletal cells plays the main role in glucocorticoid-induced osteoporosis (GIO). In GIO, glucocorticoid induces a decrease in osteoblastgenesis and an increase in the apoptosis of mature osteoblast and osteocyte, by inhibiting the expression of osteoblastic key genes, resulting in osteoblastic bone formation [12].

Immobilization induces bone loss, since the reduction of compressive mechanical forces during immobilization results in stimulating osteoclast activity by inducing bone resorption factors, such as receptor activator of NF- $\kappa$ B ligand [13]. In this patient, the long-term immobilization state might enhance bone resorption, leading to an increase of bone resorption indices in spite of glucocorticoid administration. The change of some soluble factors in the serum of this patient by glucocorticoid use and immobilization might be responsible for enhancing the level of ALP, OCN and BMP-2 in osteoblast. Furthermore, increased bone resorption indices may be due to BMP-2, stimulation of bone resorption by enhanced osteoclast formation, and osteoclastic activity [14,15]. Okamoto et al. [16] reported that mice overexpressing BMP4 in bone developed severe osteopenia with increased osteoclast number. Enhanced BMP activity itself might be one of the causes of enhanced bone resorption indices 
in this FOP patient.

The serum was obtained before taking $0.75 \mathrm{mg}$ betamethazone in the morning, $24 \mathrm{hr}$ after administration the previous day. Serum concentration of betamethasone, the next morning, was considered to be very low, since Miyachi et al. reported that betamethazone in the serum disappeared $24 \mathrm{hr}$ after oral administration, otherwise the patient developed liver failure [17]. These findings indicate that the residual betamethasone did not modulate the effects of serum from the FOP patient in the present study.

\section{Conclusion}

We presented a case of FOP with progressive ossification in extra-skeletal tissues with ACVR1 mutation. This mutation was responsible for the pathogenesis in this patient. From the present data, a systemic humoral factor may be related to the pathogenesis of FOP in this patient. Some bone anabolic factors might be elevated in the serum from the patient with FOP. Alternatively, some inhibitory factors for bone formation could be suppressed in the patient serum. Moreover, the effects of the patient serum on ALP, OCN and BMP-2 should be confirmed in several patients with FOP. Further studies are necessary to clarify this issue and will be performed in the near future.

\section{Acknowledgements}

We greatly thank Yoshiko Higashimaki and Erika Matsumoto for expert technical assistance. This work was supported in part by a grant from a grant-in-aid (21591179) from the Ministry of Science, Education, and Culture of Japan (to H.K), the Global COE Program F11 from the Ministry of Education, Culture, Sports, Science, and Technology of Japan.

\section{REFERENCES}

[1] J. M. Connor and D. A. Evans, "Fibrodysplasia Ossificans Progressiva: The Clinical Features and Natural History of 34 Patients," Journal of Bone and Joint Surgery of Britain, Vol. 64, No. 1, 1982, pp. 76-83.

[2] G. Feldman, M. Li, S. Martin, M. Urbanek, J. A. Urtizberea, M. Fardeau, M. LeMerrer, J. M. Connor, J. M. Triffitt, R. Smith, M. Muenke, F. S. Kaplan and E. M. Shore, "Fibrodysplasia Ossificans Progressiva, a Heritable Disorder of Severe Heterotopic Ossification, Maps to Human Chromosome 4q27-31," American Journal of Human Genetics, Vol. 66, 2000, pp. 128-135. doi:10.1086/302724

[3] F. H. Gannon, B. A. Valentine, E. M. Shore, M. A. Zasloff and F. S. Kaplan, “Acute Lymphocytic Infiltration in an Extremely Early Lesion of Fibrodysplasia Ossificans Progressiva," Clinical and Orthopedic Related Research, Vol. 346, 1998, pp. 19-25. doi:10.1097/00003086-199801000-00005
[4] A. B. Shafritz, E. M. Shore, F. H. Gannon, M. A. Zasloff, R. Taub, M. Muenke and F. S. Kaplan, "Overexpression of an Osteogenic Morphogen in Fibrodysplasia Ossificans Progressiva,” New England Journal of Medicine, Vol. 22, 1996, pp. 555-561. doi:10.1056/NEJM199608223350804

[5] E. A. Olmsted, F. S. Kaplan and E. M. Shore, "Bone Morphogenic Protein-4 Regulation in Fibrodysplasia Ossificans Progressiva," Clinical and Orthopedic Related Research, Vol. 408, 2003, pp. 331-343.

doi:10.1097/00003086-200303000-00044

[6] L. S. de la Pena, P. C. Billings, J. L. Fiori, J. Ahn, F. S. Kaplan and E. M. Shore, "Fibrodisplasia Ossificans Progressiva (FOP), a Disorder of Ectopic Osteogenesis, MisRegulates Cell Surface Expression and Trafficking BMPRIA,” Journal of Bone and Mineral Research, Vol. 20, No. 7, 2005, pp. 1168-1176. doi:10.1359/JBMR.050305

[7] E. M. Shore, M. Xu, G. J. Feldman, D. A. Fenstermacher, T. J. Cho, I. H. Choi, J. M. Connor, P. Delai, D. L. Glaser, M. LeMerrer, R. Morhart, J. G. Rogers, S. Roger, J. T. Triffitt, J. A. Urtizberea, M. Zasloff, M. A. Brown and F. S. Kaplan, "A Recurrent Mutation in the BMP Type I Receptor ACVR1 Causes Inherited and Sporadic Fibrodysplasia Ossificans Progressiva," Nature Genetics, Vol. 38, 2006, pp. 525-527. doi:10.1038/ng1783

[8] G. Li, H. Peng, K. Corsi, A. Usas, A. Olshanski and J. Huard, "Differential Effect of BMP4 on NIH/3T3 and C2C12 Cells: Implications for Endochondral Bone Formation," Journal of Bone and Mineral Research, Vol. 20, No. 9, 2005, pp. 1611-1623. doi:10.1359/JBMR.050513

[9] I. Hisa, Y. Inoue, G. N. Hendy, L. Canaff, R. Kitazawa, S. Kitazawa, T. Komori, T. Sugimoto, S. Seino and H. Kaji, "Parathyroid Hormone-Responsive Smad3-Related Factor, Tmem119, Promotes Osteoblast Differentiation and Interacts with the Bone Morphogenetic Protein-Runx2 Pathway,” Journal of Biological Chemistry, Vol. 286, 2011, pp. 9787-9796. doi:10.1074/jbc.M110.179127

[10] J. M. Wozney, V. Rosen, A. J. Celeste, L. M. Mitsock, M. J. Whitters, R. W. Kriz, R. M. Hewick and E. A. Wang, "Novel Regulators of Bone Formation: Molecular Clones and Activities," Science, Vol. 242, No. 4885, 1988, pp. 1528-1534. doi:10.1126/science.3201241

[11] E. Canalis, A. N. Economides and E. Gazzerro, "Bone Morphogenetic Proteins, Their Antagonists, and the Skelton,” Endocrine Reviews, Vol. 24, No. 2 2003, pp. 218-235. doi:10.1210/er.2002-0023

[12] S. Epstein, A. M. Inzerillo, J. Caminis and M. Zaidi, "Disorders Associated with Acute Rapid and Severe Bone Loss,” Journal of Bone and Mineral Research, Vol. 18, No. 12, 2003, pp. 2083-2094. doi:10.1359/jbmr.2003.18.12.2083

[13] E. Canalis and A. Giustina, "Glucocorticoid-Induced Osteoporosis: Summary of a Workshop,” Journal of Clinical Endocrinology \& Metabolism, Vol. 86, No. 12, 2001, pp. 5681-5685. doi:10.1210/jc.86.12.5681

[14] M. Kanatani, T. Sugimoto, H. Kaji, T. Kobayashi, K. Nishiyama, M. Fukase, M. Kumegawa and K. Chihara, "Stimulatory Effect of Bone Morphogenetic Protein-2 on Osteoclast-Like Cell Formation and Bone Resorbing Activity,” Journal of Bone and Mineral Research, Vol. 10, No. 
11, 1995, pp. 1681-1690. doi:10.1002/jbmr.5650101110

[15] H. Kaneko, T. Arakawa, H. Mano, T. Kaneda, A. Ogasawara, M. Nakagawa, Y. Toyama, Y. Yabe, M. Kumegawa and Y. Hakeda, "Direct Stimulation of Osteoclastic Bone Resorption by Bone Morphognetic Protein (BMP)-2 and Expression of BMP Receptors in Mature Osteoclasts," Bone, Vol. 27, No. 24, 2000, pp. 479-486. doi:10.1016/S8756-3282(00)00358-6

[16] M. Okamoto, J. Murai, H. Yoshikawa and N. Tsumaki, "Bone Morphogenetic Proteins in Bone Stimulate Osteo- clasts and Osteoblasts during Bone Development," Journal of Bone and Mineral Research, Vol. 21, No. 7, 2006, pp. 1022-1033. doi:10.1359/jbmr.060411

[17] Y. Miyachi, H. Yotsumoto, T. Kano, A. Mizuchi, T. Muto and L. Yanagibashi, "Blood Levels of Synthetic Glucocorticoids After Administration by Various Routes,” Journal of Endocrinology, Vol. 82, 1978, pp. 149-157.

doi:10.1677/joe.0.0820149 